\title{
Verification of Absorbed Dose using Diodes in External Beam Radiation Therapy Cobalt-60
}

\author{
${ }^{1}$ Nureddin. A. S. Musa, ${ }^{2}$ Pro. Mohamed Elfadil. M \\ P.O. Box 407, Khartoum, Sudan. \\ DOI: 10.29322/IJSRP.11.01.2021.p10990 \\ http://dx.doi.org/10.29322/IJSRP.11.01.2021.p10990
}

Sudan University of Science and Technology, College of Graduate Studies, Scientific Research

\begin{abstract}
This study aims to examine and verify the accuracy of entrance radiation dose it is given to the patient by using the diode system and adopt it as a quality assurance protocol. Diode response was calibrated and tested to ensure that the objective of the study was achieved by using a radiotherapy device used to treat malignant diseases, four p-type Isorad diode were tested and calibrated. The diodes measured the entrance dose in each field in D max depth and were compared with the calculated dose from TPS at D max depth for every single beam. Approximately $97.77 \%$ of the 136 measurement for the head and neck treatment were within the action level set $\pm 5 \%$. In this study the entrance dose measurements were performed for total of 45 treatment fields of head and neck cancer patients, and 22 patients. For head and neck measurement, the average discrepancy for 45 measurements was $3.23 \%$, while the corresponding standard deviation was $3.05 \%$. In our experience, we found that the diode system real time reading are easy to use and do not consume time during the measurement and give a direct measurement that limits the occurrence of error before starting the treatment and therefore they are ideal devices used to ensure the accuracy of treatment.
\end{abstract}

\section{INTRODUCTION}

$\mathrm{T}$ he radiotherapy treatment planning and delivery is a multistage process which consists of many sequential, complex steps of patient immobilization, imaging, dosed prescription, treatment planning and dose calculation, patient positioning, plan verification and dose delivery. To ensure that the delivered dose agrees with the prescribed dose at the end of the entire treatment process, it has been recommended by number of international organization that overall check of the entire process is carried out [1-6]. One of the recommended is iv-vivo dosimetry; in-vivo dosimetry has proved to be a useful tool for quality assurance in radiotherapy [7-16]. The purpose of in-vivo dosimetry is to verify that the treatment is carried out as prescribed. It is a suitable method to both monitor the treatment delivery and detect various errors early in the course of treatment. Patient's in-vivo measurements are subsequently compared to the values obtained from patient's teletherapy plan coming from the verified treatment planning system and dose calculation algorithm [17]. In the event that there are unacceptable differences in the measurements that are made to ensure the accuracy of the dose given to the patient, who was calculated using the computer system when planning, the causes that led to this are searched and addressed before starting the rest of the treatment according to the patient's treatment plan.
The tolerance and action level were set at the same initial level of $\pm 5 \%$, that action level was applied for head and neck cancer patients.

As recommended by international Commission on radiation Units and measurements ICRP has recommended that radiation dose must be delivered to within $\pm 5 \%$ of the prescribed dose [ 18 , 19]. Moreover in a recent publication by the IAEA (2013), an appropriate goal is to be able to use a tolerance level of 5\% for simple treatment, with a level of $7 \%$ for situations such as breast treatment and other treatment where measurement complications exist. However, it's recommended that, although in the initial stages of introduction of in-vivo dosimetry the tolerance levels may need to be higher, every effort should be made to achieve tolerance levels of about $5 \%$ by a process of progressive elimination of identified causes of dose differences [20].

There are a variety of detectors for verifying the delivery of the intake dose to the patient during treatment, such as titration films, TLD, MOSFET, OSL and diode, each of which has distinct characteristics and is also available for in-vivo dosimtery. In this study, the diodes were calibrated and correction factors for diodes were determined in-vivo dosimetry as described, the entrance dose represents dost at depth maximum for specific energy. The determination of calibration and correction factors was done as recommended by the ESTRO Booklet No.5 [21].

\section{MATERIALS AND METHODS}

Treatment machine and dosimetry system: All diode calibrations, correction factors determination carried out using One Cobalt-60 (CIRUS) unit were used with our in-vivo dosimetry system. A Cobalt-60 unit is used with energy of 1.25 $\mathrm{MV}$, beam radiation reaches its maximum dose at $0.5 \mathrm{~cm}$ below the skin surface; therefore, it was especially well suited for tumor within $5 \mathrm{~cm}$ of the skin surface in other parts of the body such as head and neck. The beam of $\mathrm{Co}^{60}$ machine was calibrated with ion chamber positioned at $5 \mathrm{~cm} D$ max in water phantom according to the guidelines of international Atomic Energy Agency (IAEA) TRS-398 Protocol [22].

The in-vivo dosimetry system used with diodes was IVD Model (ISORAD ${ }^{\mathrm{TM}}$ Sun Nuclear Corporation, Model p-type, USA), four diodes conducting in this study labeled as A, B, C and D), was used for measurements of entrance doses. In this study the entrance dose measurements were performed for total of (45) treatment fields of head and neck cancer patients, and (22) cancer patients, undergoing $\mathrm{H} \& \mathrm{~N}$ a number combination of treatment 
fields such as anterior posterior (AP), Left lateral (LL), Right lateral (R L) open field or with wedge fields such as anterior posterior (AP), Left lateral (LL), Right lateral (R L) open field or with wedge fields. All the patients treated with SSD technique. Often the measurement is performed in the first or second session of the start of the treatment course, after that, the action level is set $\pm 5 \%$ for entrance dose as recommended. This study was conducted and data collected after approval from the research unit of the NSCI- Libya. The diode is securely attached to the patient's surface in the central axis of the beam irradiated with time obtained by TPS for all beam use for head and neck irradiation. The readings obtained from the diode are recorded and compared to the doses that were calculated using the TPS and the percentage of difference between the calculated and received dose for the patient is calculated. Statistical package for Social Sciences t-test online was used for data analysis. The comparison of the measured and calculated doses is expressed in terms of percentage difference.

\section{RESULTS}

A total of 22 treatment cases were considered for this study, 45 treatment fields were made and monitored for head and neck cancer patients, all patients had their treatments planning with TPS.

A detailed of the in-vivo measurements on patients with the diodes are presented in Table 1 for the irradiation site. The values of mean and standard deviation of distribution of discrepancies between the measured/ expected entrance doses are presented in the Table 1 together with the percentage of measurements for which the discrepancy was within $\pm 5 \%$ tolerance level set.

Tab.2 the value of mean standard deviation and standard deviation of the distribution expected entrance dose are presented in table. 2 together with the percentage of discrepancy. The tolerance level was set $\pm 5 \%$.

\begin{tabular}{|c|c|c|c|}
\hline $\begin{array}{l}\text { No: } \\
\text { Fields }\end{array}$ & $\%$ Discrepancy & $\begin{array}{l}\text { Standard deviation } \\
\text { SD\% }\end{array}$ & $\begin{array}{l}\text { Mean } \\
\text { SD \% }\end{array}$ \\
\hline 1 & 7 & 4.56 & 3.23 \\
\hline 2 & 6 & 4.16 & 2.94 \\
\hline 3 & 7 & 8.53 & 6.03 \\
\hline 4 & 5 & 5.82 & 4.11 \\
\hline 5 & 8 & 9.68 & 6.84 \\
\hline 6 & 0.3 & 0.2 & 0.14 \\
\hline 7 & 0.7 & 0.41 & 0.29 \\
\hline 8 & 8 & 8.63 & 6.1 \\
\hline 9 & 1.7 & 0.92 & 0.65 \\
\hline 10 & 7 & 3.87 & 2.74 \\
\hline 11 & 2.1 & 1.21 & 0.85 \\
\hline 12 & 1.2 & 0.67 & 0.47 \\
\hline 13 & 0.5 & 0.36 & 0.25 \\
\hline 14 & 1.4 & 1.01 & 0.71 \\
\hline 15 & 4.5 & 4.56 & 3.22 \\
\hline 16 & 6 & 6.52 & 4.61 \\
\hline 17 & 5 & 2.3 & 1.62 \\
\hline 18 & 2 & 1.86 & 1.31 \\
\hline 19 & 7 & 4.51 & 3.19 \\
\hline 20 & 1.9 & 1.27 & 0.89 \\
\hline
\end{tabular}




\begin{tabular}{|l|l|l|l|}
\hline 21 & 1.8 & 1.12 & 0.79 \\
\hline 22 & 2.5 & 1.75 & 1.24 \\
\hline 23 & 1.8 & 1.23 & 0.86 \\
\hline 24 & 14 & 8.95 & 6.33 \\
\hline 25 & 0.2 & 0.12 & 0.08 \\
\hline 26 & 0.2 & 0.125 & 0.08 \\
\hline 27 & 4 & 2.67 & 1.89 \\
\hline 28 & 1 & 0.67 & 0.47 \\
\hline 29 & 2.3 & 1.67 & 1.18 \\
\hline 30 & 1 & 0.67 & 0.47 \\
\hline 31 & 0.1 & 0.07 & 0.05 \\
\hline 32 & 1.3 & 1.22 & 0.86 \\
\hline 33 & 3.5 & 1.63 & 1.15 \\
\hline 34 & 0.8 & 0.83 & 0.58 \\
\hline 35 & 9 & 9.46 & 6.68 \\
\hline 36 & 1.2 & 0.85 & 0.60 \\
\hline 37 & 0.3 & 0.23 & 0.16 \\
\hline 38 & 6 & 6.57 & 4.64 \\
\hline 39 & 4 & 4.42 & 3.1 \\
\hline 40 & 0.3 & 0.2 & 0.14 \\
\hline 41 & 0.6 & 0.36 & 0.25 \\
\hline 42 & 0.1 & 0.06 & 0.04 \\
\hline 43 & 0.5 & 0.52 & 0.36 \\
\hline 44 & 3.4 & 2.14 & 1.51 \\
\hline 45 & 3.1 & 1.98 & 1.40 \\
\hline & & & \\
\hline
\end{tabular}

The mean value of the standard deviation distributions $3.2289 \pm 0.455$ for all measurements with errors was \pm 0.455 and the standard deviation was $3.05 \%$. The standard deviations within action level $\pm 5 \%$ were detected in all measurements.

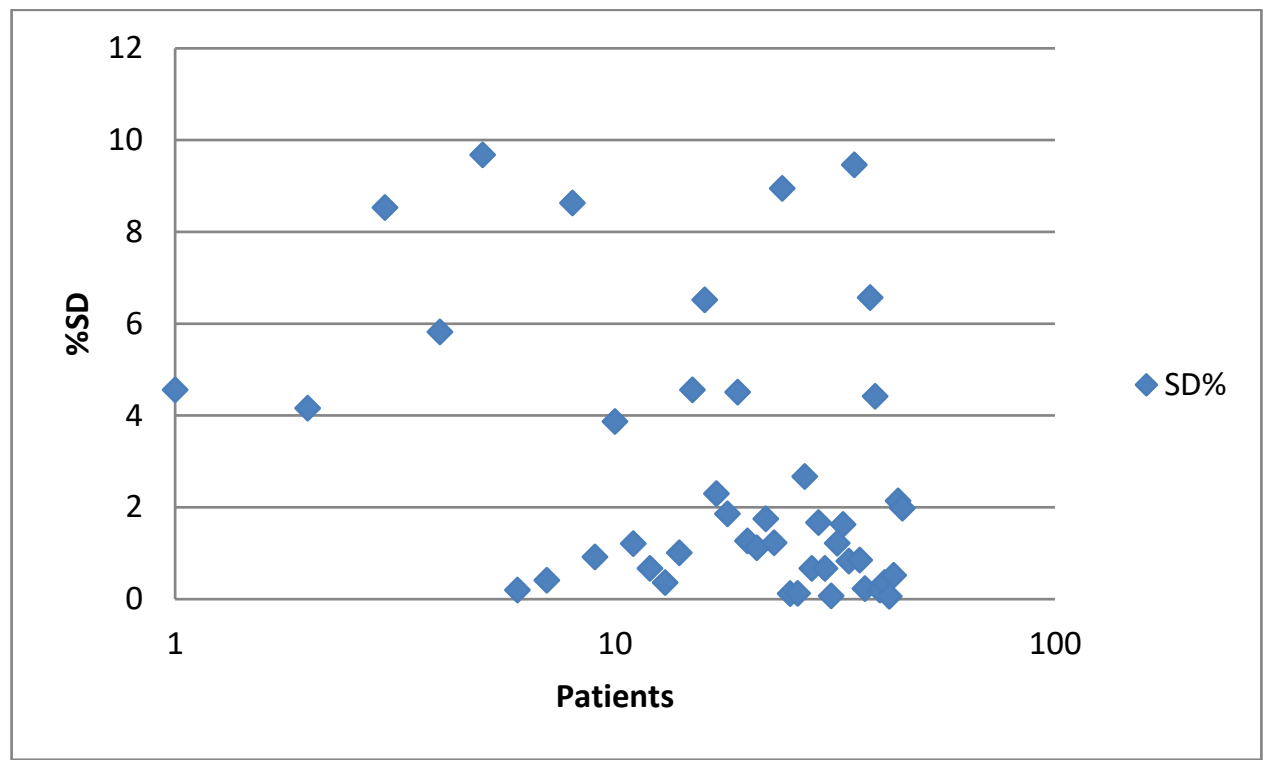

Figure.1 distribution value of standard deviation expected entrance dose for head and neck.

\section{DISCUSSION}

Based on the characteristics of the diode in-vivo, it is easy to use and does not consume time during the measurement, and it gives a direct measurement reading that reduces the occurrence of error before starting treatment and can be used to calculate the patient's entrance and exit dose compared to other types used in the same target.

In-vivo dosimetry results for patients with head and neck cancer have shown better. Out of the 45 fields measurements made for the head and neck, one (1) fields had discrepancies outside the $\pm 5 \%$ action level set. As indicate in table (2) approximately 
$97.77 \%$ of the 45 measurements for head and neck treatments were within the $\pm 5 \%$ action level, one (1) measurements discrepancies outside the action level was recorded for this measurement, the average discrepancy for 45 measurements $(\mathrm{N})$ was $3.23 \% \pm 0.455$ while the corresponding standard deviation was $3.05 \%$.

The analysis of 45 measurements showed $97.77 \%$ accuracy in dose delivery, and delivered on overall mean of $3.23 \%$, and the standard deviation of $3.05 \%$. This indicates that the combined uncertainty of the treatment delivery and in-vivo dosimetry at the NSCI- Libya is $3.05 \%$. Discrepancies exceeding $10 \%$ are immediately should be reviewed before treatment starts The results for diode were found to be similar to other results in literature [18, 19], also similar to results founded by Gadhi, M, A et al [22].

\section{CONCLUSION}

In summary, in our experience, we found that the diode system (real time) are easy to use and do not consume time during the measurement and give a direct measurement reading that limits the occurrence of error before starting the treatment and therefore they are ideal devices used to ensure the accuracy of treatment., Therefore, it is ideal in terms of use to verify the accuracy of the therapeutic dose given to the patient in-vivo dosimetry is an effective method for detecting radiotherapy errors and fulfilling requirements set forth by national and international regulations.

\section{RECOMMENDATION}

- We recommend that the diode positioning on a patient's skin and the angular diode sensitivity be reconsidered.

- We also recommended that a more accurate calculation of expected diode values be performed especially for fields that pass through the treatment table these efforts would enable the achievement of action levels of $\pm 5 \%$

- We recommend mistakes that occur should be documented and studied to avoid them not happening again.

- We recommend further studies to calculate the patient's exit/ middle line dose as well as critical organ dose and skin dose.

\section{APPENDIX A: Table 1: The comparison of the calculated dose, measured dose and percentage of difference between both.}

\begin{tabular}{|l|l|l|l|}
\hline $\begin{array}{l}\text { No: } \\
\text { Fields }\end{array}$ & $\begin{array}{l}\text { Calculated dose } \\
\text { (cGy) }\end{array}$ & $\begin{array}{l}\text { Measured dose } \\
\text { (cGy) }\end{array}$ & $\begin{array}{l}\% \\
\text { Difference }\end{array}$ \\
\hline 1 & 132.56 & 141.7 & 7 \\
\hline 2 & 132.56 & 140.88 & 6 \\
\hline 3 & 228.89 & 245.95 & 7 \\
\hline 4 & 228.89 & 240.54 & 5 \\
\hline 5 & 228.89 & 248.25 & 8 \\
\hline 6 & 127.5 & 127.1 & 0.3 \\
\hline 7 & 127.5 & 128.33 & 0.7 \\
\hline 8 & 226.38 & 243.64 & 8 \\
\hline 9 & 109.97 & 108.12 & 1.7 \\
\hline
\end{tabular}

This publication is licensed under Creative Commons Attribution CC BY.

\begin{tabular}{|c|c|c|c|}
\hline 10 & 109.97 & 117.72 & 7 \\
\hline 11 & 116.37 & 113.95 & 2.1 \\
\hline 12 & 116.37 & 117.72 & 1.2 \\
\hline 13 & 147.33 & 148.05 & 0.5 \\
\hline 14 & 147.33 & 149.35 & 1.4 \\
\hline 15 & 202.26 & 211.39 & 4.5 \\
\hline 16 & 202.88 & 215.92 & 6 \\
\hline 17 & 89.32 & 84.72 & 5 \\
\hline 18 & 178.64 & 174.92 & 2 \\
\hline 19 & 130.61 & 121.58 & 7 \\
\hline 20 & 130.61 & 128.07 & 1.9 \\
\hline 21 & 128.20 & 130.45 & 1.8 \\
\hline 22 & 139.2 & 142.71 & 2.5 \\
\hline 23 & 139.2 & 141.66 & 1.8 \\
\hline 24 & 125.69 & 107.78 & 14 \\
\hline 25 & 125.69 & 125.94 & 0.2 \\
\hline 26 & 133.32 & 133.57 & 0.2 \\
\hline 27 & 133.32 & 127.97 & 4 \\
\hline 28 & 144.40 & 145.75 & 1 \\
\hline 29 & 144.40 & 147.75 & 2.3 \\
\hline 30 & 144.40 & 143.06 & 1 \\
\hline 31 & 144.40 & 144.56 & 0.1 \\
\hline 32 & 186.96 & 184.52 & 1.3 \\
\hline 33 & 93.48 & 90.21 & 3.5 \\
\hline 34 & 217.31 & 215.65 & 0.8 \\
\hline 35 & 217.31 & 236.23 & 9 \\
\hline 36 & 144.25 & 145.96 & 1.2 \\
\hline 37 & 144.25 & 144.71 & 0.3 \\
\hline 38 & 216.23 & 203.09 & 6 \\
\hline 39 & 216.23 & 207.38 & 4 \\
\hline 40 & 129.41 & 129.01 & 0.3 \\
\hline 41 & 129.41 & 130.14 & 0.6 \\
\hline 42 & 222.8 & 222.93 & 0.1 \\
\hline 43 & 222.8 & 221.76 & 0.5 \\
\hline 44 & 127.77 & 123.48 & 3.4 \\
\hline 45 & 127.77 & 123.80 & 3.1 \\
\hline
\end{tabular}

\section{REFERENCES}

[1] [1]- Diode in vivo Dosimetry for Patients Receiving External Beam Radiation Therapy, AAPM, Task Group 62, Report No.87, M.P. Publishing, Madision, Wis, U.S.A, 2005.

[2] [2]- Determination of Absorbed Dose in a Patient Irradiation by Beam of X of Gamma Rays in Radiotherapy Procedures, ICRU Report 24, Washington DC, U.S.A, 1976.

[3] [3]- Quality Assurance in Radiotherapy, WHO, Genev, 1988.

[4] [4]-Procedures in External Radiation Therapy Dosimetry with Electron and Photon Beams with Maximum Energies between $1 \mathrm{MeV}$ and $50 \mathrm{MeV}$, Nordic association of Clinical Physics(NACP), Acta Radiol.Oncol., 19(1980), 1, pp.55-79.

[5] [5]- Comprehensive Audits of Radiotherapy Practices: a Team for Radiation Oncology (QUATRO), Intenational Atomic Energy Agency, Vienna, 2007.

[6] [6]- Setting Up a Radiotherapy Programme: Clinical, Medical Physics, Radiation Protection and Safety Aspects,: International Atomic Energy Agency, Vienna, 2008.

[7] [7]- Fontela, D.P., et al., Customization of a Radiation Management System to Support in-vivo Patient Dosimetry Using Diodes, Med.Phy., 23(1996),8, pp1425-1429. 
[8] [8]- Fontela, D.P., et al., The Use of Diode Dosimtery in Quality Improvement of Patient Care in Radiation Dosimetry, Med. Dosim., 21(1996), 4,pp. 235-241.

[9] [9]- Heukelom, S., Lanson ,J. H., Mijnheer, B.J., Comparison of Entrance and Exit Dose Measurements Using Ionization Chambers and Silicon Diodes, Phys. Med. Biol., 36 (1991), 1 ,pp. 47 -59.

[10] [10]- Heukelom, S., Lanson ,J. H., Mijnheer, B.J., in-vivo Dosimetry During Pelvic Treatment, Radiother. Oncl., 25 (1992), 2, pp.111-120.

[11] [11]- Heukelom, S., et al., In vivo Dosimetry During Tangential Breast Treatment, Radiother. Oncol., 22(1991), 4, pp. 269-279.

[12] [12]- Lenunens, G., et al., Quality Assurance in Radiotherapy by in-vivo Dosimetry, 1 Entrance Dose Measurements, a Reliable Procedure, Radiother. Oncol., 17(1990), 2,pp. 141-151.

[13] [13]-Leunens, G., et al., Quality assurance in Radiotherapy by absorbed Dose, Radiother, Oncol., 19(1990), 1, pp.73-87.

[14] [14]- Li.Ch., Lamel, L.S., Tom, D., A patient Dose Verification Program Using Diode Detectors, Med. Dosim., 20(1995), 3,pp. 209-214.

[15] [15]- Meiler, R.J., Podgorsak, M.B., Characterization of the Response of Commercial Diode Detectors Used for in-vivo Dosimetry, Med. Dosim., 22(1997), 1, pp. 31-37.

[16] [16]- Essers, M., Mijnheer. B.J., In vivo Dosimetry During External Photon Beam Radiotherapy, Int. J. Radiat. Oncol. Biol. Phys., 43 (1999), 2, pp. 245 259.

[17] [17]- Rutonjski, L., et al., Dosimetric Verification of Radiotherapy Treatment Planning Systems in Serbia:National Audit, Radiation Oncology , (2012), Art.No155.

[18] [18]- Alam R, IRbbott GS, Pourang R, Nath R. Application of AAPM Radiation therapy Committee task Group 23 test package for comparision of two treatment planning systems for photon external beam radiotherapy. Med Phys 1997; 24:2043-54.

[19] [19]- International commission on radiation units and measurements ICRU. Dose specifications for reporting external beam therapy with photons and electrons. ICRP Report 29, Baltimore, MD: ICRU Bethesds, MD, 1978.

[20] [20]- IAEA HUMAN HEALTH REPORT No.8. Development for procedures for in-vivo dosimetry in Radiotherapy. Pp 31-40, 2013

[21] [21]- Huyskens, D.P., et al., Practical Guidelines for the Implementation of in vivo Dosimetry with Diodes in External Radiotherapy with Photon Beams (Entrance Dose), ESTRO Booklet No.5.

[22] [22]- Gadhi, M, A., et al ., Radiation oncology. Dosimetry . Absorbed dose. Diode. Entrance/ exit dose . Ionizion radition, Ausralas Phys Eng Sci Med (2016) 39:211-219.

\section{AUTHORS}

First Author - Nureddin. A. S. Musa, Sudan University of Science and Technology, College of Graduate Studies, Scientific Research, .O. Box 407, Khartoum, Sudan., nureddine2001@yahoo.com

Second Author - Pro. Mohamed Elfadil. M, Sudan University of Science and Technology, College of Graduate Studies, Scientific Research, .O. Box 407, Khartoum, Sudan., mohamedelfadilmohamed@gmail.com 